\title{
Left ventricular pseudoaneurysm in a patient with Dressler's syndrome after myocardial infarction
}

\author{
H Reinecke, $\mathrm{T}$ Wichter, $M$ Weyand
}

Department of

Cardiology and

Angiology, Hospital of the Westfälische

Wilhelms-University,

Münster, Germany

H Reinecke

T Wichter

Department of

Thoracic and

Cardiovascular

Surgery, Hospital of

the Westfälische

Wilhelms-University

$M$ Weyand

Correspondence to:

Dr H Reinecke,

Medizinische Klinik und

Poliklinik-Innere Medizin

C, Westfälische

Wilhelms-Universität,

D-48129 Münster, Germany.

23 December 1997
Accepted for publication

\section{Abstract}

Successful recanalisation of the left anterior descending coronary artery was performed in a 51 year old man who was admitted two weeks after acute anterior myocardial infarction. Fourteen days later, the patient developed Dressler's syndrome with cardiac tamponade, which was immediately punctured. Sternotomy was performed after two weeks because of progressive haemodynamic deterioration, and fibrinous masses were removed from the pericardium. The patient recovered but two weeks later echocardiography showed a perforation of the left ventricular free wall and formation of a pseudoaneurysm. Intensive monitoring showed significant enlargement of the pseudoaneurysm, which was subsequently resected. This case demonstrates that dangerous formation of a pseudoaneurysm can occur not only during the first days of acute myocardial infarction but also after weeks in patients suffering from non-infectious pericarditis caused by Dressler's syndrome. Although the incidence of Dressler's syndrome is declining, patients

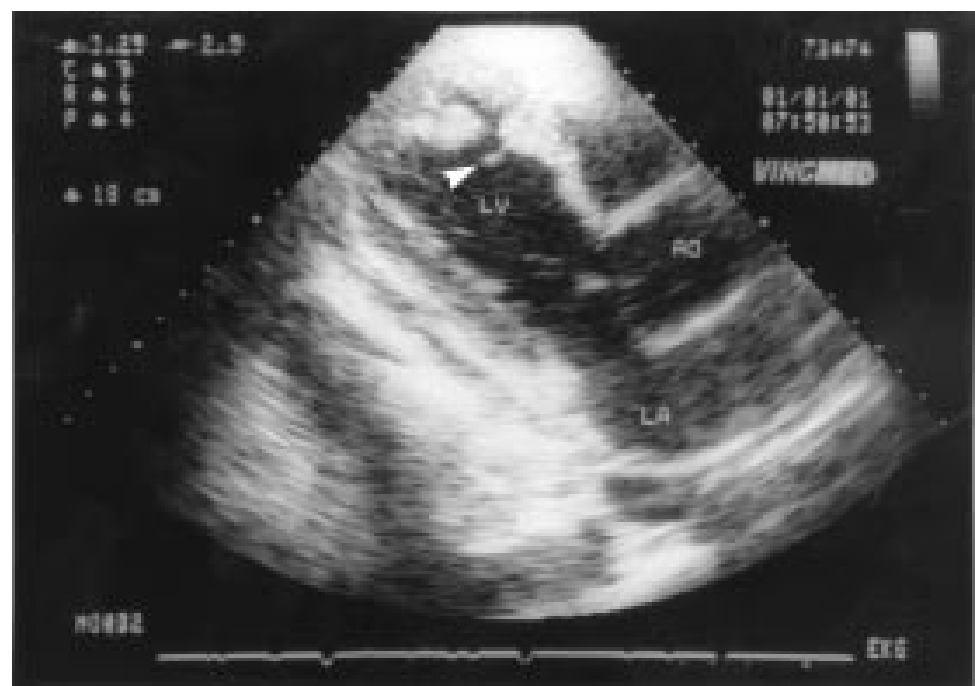

Figure 1 Four weeks after the onset of Dressler's syndrome (eight weeks after myocardial infarction) the pseudoaneurysm was primarily detected. Echocardiographic three chamber view shows perforation of the left ventricular wall, extracardial space, and floating parts of myocardial tissue at the margins of the perforation (arrow). LA, left atrium; LV, left ventricle; $A O$, aorta. should be monitored carefully for several weeks, especially by echocardiography. (Heart 1998;80:98-100)

Keywords: Dressler's syndrome; pseudoaneurysm; myocardial infarction

Perforation or rupture of the left ventricular free wall is a dramatic and often fatal complication that typically occurs three to five days after the onset of acute myocardial infarction (AMI). Adhesions of epicardium and pericardium, and a parietal thrombus at the region of infarct may lead to the formation of a pseudoaneurysm in which the walls are formed by pericardium and organised thrombotic material. ${ }^{1}$ Because of a high incidence of spontaneous and frequently fatal ruptures of these pseudoaneurysms, the treatment of choice is surgical resection.

In addition to this acute complication, Dressler's syndrome (also called postmyocardial infarction syndrome) can occur two to eight weeks after the onset of AMI. ${ }^{2}$ This complication was originally described as occurring in $1-4 \%$ of all patients with AMI. ${ }^{3}$ Characteristics are systemic signs of inflammation (malaise, fever, leucocytosis) without evidence of infection, together with pericardial and often pleural effusion. Cardiac tamponade is a rare but dangerous manifestation of the syndrome. ${ }^{2}$ Although the pathogenesis of Dressler's syndrome is not clear, an immunopathological genesis has been considered. ${ }^{2-4}$

We describe a patient who developed left ventricular pseudoaneurysm four weeks after onset of exudative pericarditis associated with Dressler's syndrome, who had successful surgical resection.

\section{Case report}

A 51 year old man developed a large anterior myocardial infarction with a peak creatine kinase of $1700 \mathrm{U} / 1$ despite thrombolysis. Fourteen days after onset of the infarction he was admitted to our department for coronary angiography because of unstable angina pectoris. Angiography showed mild dyskinesia of the anterior wall and single vessel disease with a proximal occlusion of the left anterior descending coronary artery. Subsequent recanalisation with angioplasty was successful. Two weeks 

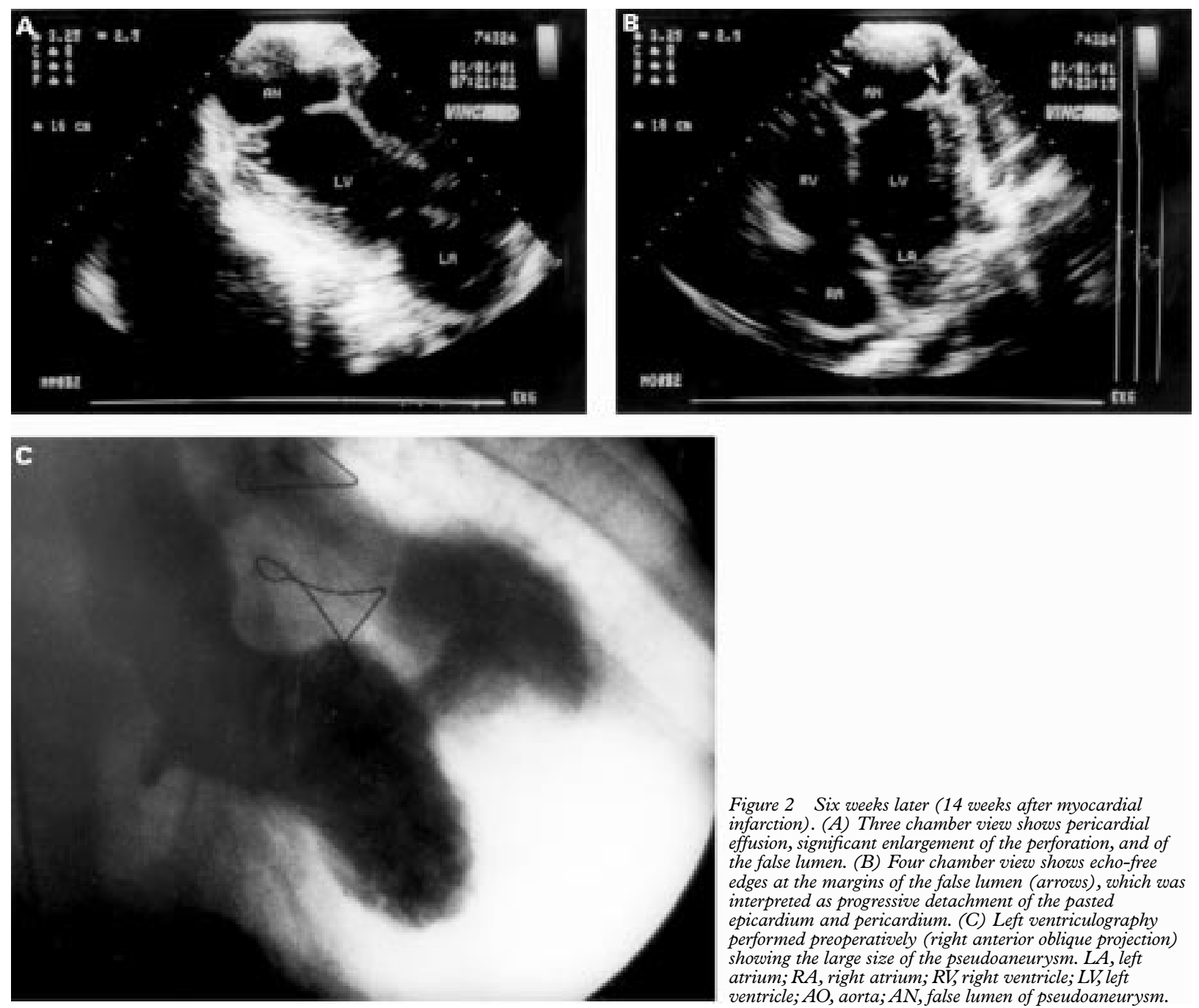

Figure 2 Six weeks later (14 weeks after myocardial infarction). (A) Three chamber view shows pericardial effusion, significant enlargement of the perforation, and of the false lumen. (B) Four chamber view shows echo-free edges at the margins of the false lumen (arrows), which was interpreted as progressive detachment of the pasted epicardium and pericardium. (C) Left ventriculography performed preoperatively (right anterior oblique projection) showing the large size of the pseudoaneurysm. LA, left atrium; $R A$, right atrium; $R V$, right ventricle; $L V$, left ventricle; $A O$, aorta; $A N$, false lumen of pseudoaneurysm.

after recanalisation (four weeks after AMI), the patient developed fever and showed signs of cardiac decompensation with tachypnoea, hypotension, and sinus tachycardia. At this time, echocardiography showed cardiac tamponade and dyskinesia of the anterior wall. An emergent pericardial puncture was performed; $720 \mathrm{ml}$ of serous liquid were drained from the pericardium and the patient recovered haemodynamically. The drained fluid was sterile and contained a significant amount of polymorphonuclear leucocytes. Over the next two weeks, $100 \mathrm{ml} /$ day of sterile pericardial effusion was drained, and the patient had persistent signs of general infection: blood leucocytosis of 20000 leucocytes $/ \mu \mathrm{l}$ and continuous fever $>38.5^{\circ} \mathrm{C}$. Chest radiography showed mild bilateral pleural effusion. The patient worsened haemodynamically and echocardiography showed echorich masses surrounding the heart. A sternotomy was performed during which significant masses of fibrinous detritus were removed and three drains inserted for pericardial lavage. No infectious agent was isolated in several blood samples or the material from the sternotomy, nevertheless prophylactic antibiotic treatment was started. Postoperatively, the patient felt well and after two weeks he showed no more clinical signs of inflammation.
Four weeks after onset of pericarditis (eight weeks after AMI), routine echocardiography showed a perforation of the anterior left ventricular wall (fig 1) with small, floating parts of myocardial tissue at the margins of the perforation. Colour Doppler showed a flow between the left ventricular cavity and the "false lumen" (not shown). A parietal thrombus was also observed but dissolved after one week with anticoagulation using heparin. Asymptomatic myocardial re-infarction as a possible cause for the perforation was excluded because of daily electrocardiographic and laboratory measurements. Surgery was postponed because of the recent active pericarditis. Intensive echocardiographic controls for the next six weeks showed mild but progressive pericardial effusion, significant enlargement of the wall perforation and of the false lumen (figs 2A and B). Additionally, echo-free separations appeared at the margins of the false lumen and became larger. This was interpreted as progressive detachment of the pasted epicardium and pericardium (fig 2B). A preoperative coronary angiography showed a good short term result of angioplasty, and left ventriculography showed the large size of the pseudoaneurysm (fig 2C). 
A repeat sternotomy was performed using extracorporeal circulation and cold cardioplegic cardiac arrest (six weeks after diagnosis of the perforation). The false lumen was freed from its surrounding adhesions and partially resected, including the rest of the parietal thrombotic material. The borders of the perforated defect were sewed and armed with a Dacron patch. The free margins of the false lumen were covered over the closed perforation and fixed as an additional enforcement. Further postoperative follow up was free from complications, and the patient was discharged to a rehabilitation centre two weeks after surgery.

\section{Discussion}

In addition to the reports about pseudoaneurysms as a typical early complication after AMI, there have been a few reports of pseudoaneurysm formation in patients without coronary heart disease caused by purulent pericarditis ${ }^{5}$ (even in a child ${ }^{6}$ ), endocarditis, and penetrating chest trauma. ${ }^{8}$

The present case reports a combination of two typical complications after AMI, which to our knowledge have not been reported before. Four weeks after AMI, our patient developed systemic signs of inflammation and cardiac tamponade. The leucocyte rich but sterile pericardial effusion and the fibrinous detritus later removed from the pericardium are typical signs of Dressler's syndrome. ${ }^{2}$ Perforation of the left ventricular anterior wall and formation of a pseudoaneurysm were discovered, which were obviously induced by the exudative and non-infectious pericarditis associated with Dressler's syndrome.

Treatment with glucosteroids and nonsteroidal anti-inflammatory agents has been recommended for the management of patients with Dressler's syndrome. ${ }^{3}$ However, there have been reports that such treatment after AMI increases the risk of ventricular rupture with possible subsequent formation of pseudoaneurysm. ${ }^{9}$ Our patient developed the pseudoaneurysm without receiving any of these drugs.

The prevalence of Dressler's syndrome was originally thought to be $1-4 \%$ of patients with AMI. ${ }^{3}$ Recent studies have shown the incidence to be declining because of successful thrombolysis in patients with AMI. ${ }^{10}$ Although the incidence has decreased, and formation of pseudoaneurysm after Dressler's syndrome has to be considered a rare complication, the high rate of spontaneous and often fatal ruptures of pseudoaneurysms should be remembered. Because prompt diagnosis and surgical treatment are essential, an intensive and prolonged monitoring of patients with Dressler's syndrome should be performed, particularly with echocardiography.

1 Brown SL, Gropler RJ, Harris KM. Distinguishing left ventricular aneurysm from pseudoaneurysm: a review of the literature. Chest 1997;111:1403-9.

2 Gregoratos G. Pericardial involvement in acute myocardial infarction. Cardiol Clin 1990;8:601-8.

3 Kahn A. Pericarditis of myocardial infarction: review of the literature with case presentation. Am Heart f 1975;90:78894.

4 Earis JE, Marcuson EC, Bernstein A. Complement activation after myocardial infarction. Chest 1985;87:18690.

5 Wiegers SE, Plehn JF, Rajail-Khorasami A, et al. Purulent pericarditis and ventricular pseudoaneurysm in an intravenous drug abuser. Am Heart $\mathcal{F}$ 1988;116:1635-7.

6 Pahl MMC, Arruda J, Gillio AE, et al. Left ventricular pseudoaneurysm: an unusual complication of pericarditis and Staphylococcus aureus septicemia. Pediatr Infect Dis $\mathcal{F}$ 1996;15:380-2.

7 Kessler KM, Kieval J, Saksena S, et al. Echocardiographic features of posterior left ventricular wall pseudoaneurysm due to Escherichia coli endocarditis. Am Heart $71982 ; 103$ : due to Esch-42.

8 Matsumoto AY, Ortiz J, Nanda NC. Left ventricular pseudoaneurysm due to penetrating injury of the chest: an echocardiographic diagnosis. Am Heart f 1988;115:1134-

9 Silverman HS, Pfeifer MP. Relation between use of anti-inflammatory agents and left ventricular free wall rupture during acute myocardial infarction. Am $f$ Cardiol 1987;59:363-4.

10 Shahar A, Hod H, Barabash GM, et al. Disappearance of a syndrome: Dressler's syndrome in the era of thrombolysis. Cardiology 1994;85:255-8. 